\title{
Patient Privacy in the Era of Big Data
}

\author{
Mehmet Kayaalp
}

National Library of Medicine, National Institutes of Health, Maryland, ABD

Privacy was defined as a fundamental human right in the Universal Declaration of Human Rights at the 1948 United Nations General Assembly. However, there is still no consensus on what constitutes privacy. In this review, we look at the evolution of privacy as a concept from the era of Hippocrates to the era of social media and big data. To appreciate the modern measures of patient privacy protection and correctly interpret the current regulatory framework in the United States, we need to analyze and understand the concepts of individually identifiable information, individually identifiable health information, protected health information, and de-identification. The Privacy Rule of the Health Insurance Portability and Accountability Act defines the regulatory framework and casts a balance between protective measures and access to health information for secondary (scientific) use. The rule defines the conditions when health information is protected by law and how protected health information can be de-identified for secondary use. With the advents of artificial intelligence and computational linguistics, computational text de-identification algorithms produce de-identified results nearly as well as those produced by human experts, but much faster, more consistently and basically for free. Modern clinical text de-identification systems now pave the road to big data and enable scientists to access de-identified clinical information while firmly protecting patient privacy. However, clinical text de-identification is not a perfect process. In order to maximize the protection of patient privacy and to free clinical and scientific information from the confines of electronic healthcare systems, all stakeholders, including patients, health institutions and institutional review boards, scientists and the scientific communities, as well as regulatory and law enforcement agencies must collaborate closely. On the one hand, public health laws and privacy regulations define rules and responsibilities such as requesting and granting only the amount of health information that is necessary for the scientific study. On the other hand, developers of de-identification systems provide guidelines to use different modes of operations to maximize the effectiveness of their tools and the success of de-identification. Institutions with clinical repositories need to follow these rules and guidelines closely to successfully protect patient privacy. To open the gates of big data to scientific communities, healthcare institutions need to be supported in their deidentification and data sharing efforts by the public, scientific communities, and local, state, and federal legislators and government agencies.

Keywords: Health Insurance Portability and Accountability Act, medical informatics, confidentiality, data anonymization data sharing, personally identifiable information, privacy

Whatsoever I shall see or hear in the course of my profession, ...

I will never divulge, holding such things to be holy secrets,

-Hippocrates (1).

\footnotetext{
Address for Correspondence: Dr. Mehmet Kayaalp, National Library of Medicine, National Institutes of Health, Maryland, ABD Phone: 301-827-5017 e-mail: mehmet.kayaalp@nih.gov ORCID ID: orcid.org/0000-0003-0930-2761 Received: 17 July $2017 \quad$ Accepted: 11 September 2017•DOI: 10.4274/balkanmedj.2017.0966

Available at www.balkanmedicaljournal.org

Cite this article as:

Kayaalp M. Patient Privacy in the Era of Big Data. Balkan Med J 2018;35:8-17

${ }^{\circ}$ Copyright 2018 by Trakya University Faculty of Medicine / The Balkan Medical Journal published by Galenos Publishing House.
} 


\section{Evolution of privacy}

Privacy and confidentiality have been two of the major pillars of medical ethics since Classical Antiquity, albeit they may not always have been expressed in those terms (2). The definition and the extent of privacy have been a constant struggle for scholars and philosophers, which is still true today $(3,4)$. In the old days, it simply implied secrets of a person. In the early modern period, with the concept of my home is my castle, privacy enveloped the personal space. The Attorney General of England Sir Edward Coke stated it in 1604 as "The house of every one is to him as his castle and fortress, as well for his defence against injury and violence as for his repose" (5). This idea found it's home in the U.S. Constitution with the Fourth Amendment in 1791 as "The right of the people to be secure in their persons, houses, papers, and effects, against unreasonable searches and seizures,..." (6).

In 1890, Warren and Brandeis (7) defined privacy as "the right to be let alone," which is still in use by various contemporary authors (8-10). In 1948, United Nations General Assembly adopted the Universal Declaration of Human Rights and enshrined privacy as a fundamental human right: "No one shall be subjected to arbitrary interference with his privacy, family, home or correspondence, nor to attacks upon his honour and reputation. Everyone has the right to the protection of the law against such interference or attacks" (11). However, there was still no consensus on what constituted privacy or on its extent or limits $(3,4,12-16)$.

As our lives get more complex, the concept has become more involved and complicated. Some defined privacy in terms of solitude $(7,17)$ or control accessibility to oneself $(3)$, anonymity (4), autonomy $(2,18,19)$ or control over one's own body and sexuality $(3,4)$. With the advent of digital communication revolution, as social media becomes ubiquitous, the concept of privacy has evolved organically. Today, we define it as the right to maintain control over personal information (20), which is information about oneself, including information about one's possessions, communications, conducts, and other affairs. Physical intrusion into the personal space or obstruction of personal conducts may co-occur with the invasion of privacy but physical aspects of such breaches are defined within the realms of other civil liberties; whereas, the right to privacy provides us the legal and ethical authority to determine how and with whom our personal information can be shared.

Despite being a fundamental human right, privacy is neither absolute nor unconditional; it is limited by the rights of others and by civic duties. For example, no person can choose not to disclose their income information from the government revenue services on the grounds of privacy, but the government agency cannot disclose such personal information to others without the person's permission or without a court order. Although income tax is held as private information in the US, this is not true everywhere in the world (21) as the understanding of what is private, what needs to be disclosed to the public, and where lies the boundary between public interest and privacy differ among cultures.

\section{Protection of health information containing patient identifiers}

In the United States, any biomedical study that includes personal identifiers of an individual who is the subject of the information is categorized as human subjects research $(22,23)$. Health information (HI) is defined as information related to the past, present, and future health care or health status of the individual or information related to health care payments. Individually identifiable health information is a subset of $\mathrm{HI}$ (24) and contains identifiers or other such information that can be used to identify the subject of the health information (25). Most of the individually identifiable health information are protected health information (PHI) the main exceptions are those found in education records (e.g., immunization records) not maintained by a healthcare provider (26), in employment records, and health information of individuals deceased more than 50 years ago. Health information of the individual deceased within the last 50 years is considered PHI (25).

Individually identifiable information, also known as personally identifying information (PII), is frequently confused with individually identifiable health information. Some PII elements such as personal names and social security numbers can be found in medical records, but they are not health information, hence not PHI. We consider PHI as the set at the intersection of health information and PII (Figure 1). Although this particular set representation of $\mathrm{PHI}$ is true, it can be misinterpreted as PII in health records unless we elaborate on constituents of these sets and their relations.

In Figure 1a, $C$ is the set of all elementary (noncompound) clinically pertinent information and $P I D$ is the set of all elementary personal identifiers. These two sets overlap because some PID elements (e.g., age, gender, and ethnicity) entail clinically pertinent information. Since the set of elementary information at the intersection of $C$ and PID is mostly demographic in nature, it is labeled as $D$, but $D$ also contains some non-demographic PII such as medical record numbers. All other elements of PID such as personal names and telephone numbers comprise the set $P$. The set of health information $H$ consists of all clinically pertinent information elements that are not in $D$ (e.g., "has Parkinson's disease").

Elements of the sets $H, D$, and $P$ in Figure 1a are denoted with the corresponding lower case letters and distinguished from each other with distinct suffixes (Figure 1b). Any single health record such as $R_{1}$ in Figure 1a-c may include a 
particular combination of these information elements. If a record such as $R_{2}$ in Figure 1a is a subset of $H$ only, it would comprise health information not linked to an individual; thus, it would not constitute PHI (see the tan area in Figure 1c). If a document such as $R_{3}$ in Figure 1a contains PID elements with no elements of $H$ (e.g., a table of names and addresses), it would not be considered as health information (see the pink area of Figure 1c). Note that each element of the sets in Figure

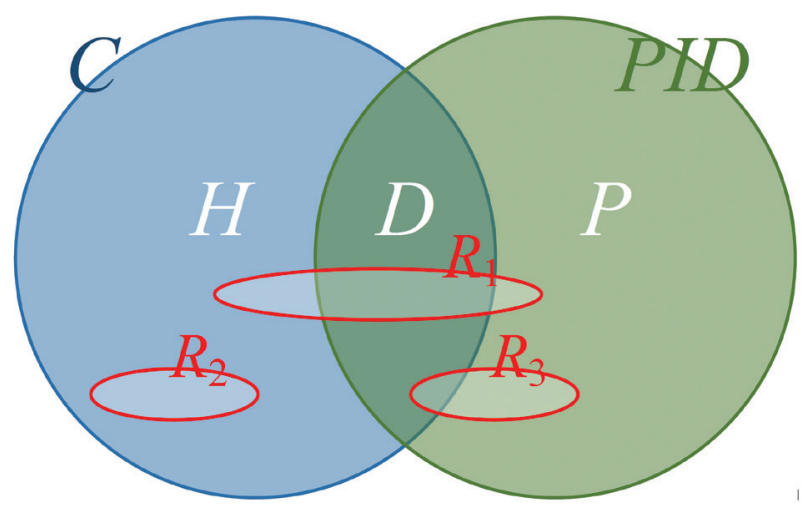

FIG. 1a. Relationship between the set $C$ of all elementary clinical information and the set PID of all elementary personal identifiers, Their subsets are health information with no personal identifiers $H$, demographic information and clinical personal identifiers $D$, non-clinical personal identifiers $P$, and three hypothetical records $R_{1}, R_{2}$, and $R_{3}$.

$$
\begin{aligned}
& H=\left\{h_{1}, h_{2}, \cdots\right\}, D=\left\{d_{1}, d_{2}, \cdots\right\}, P=\left\{p_{1}, p_{2}, \cdots\right\} \\
& R_{1}=\left\{h_{3}, \cdots, h_{91}, d_{4}, \cdots, d_{19}, p_{1}, \cdots, p_{7}\right\} \\
& R_{2}=\left\{h_{2}, \cdots, h_{115}\right\}, R_{3}=\left\{d_{3}, d_{29}, p_{3}, \cdots, p_{97}\right\}
\end{aligned}
$$

FIG. 1b. Relationship among $H, D$, and $P$ and the representation of three hypothetical records using the set notation.

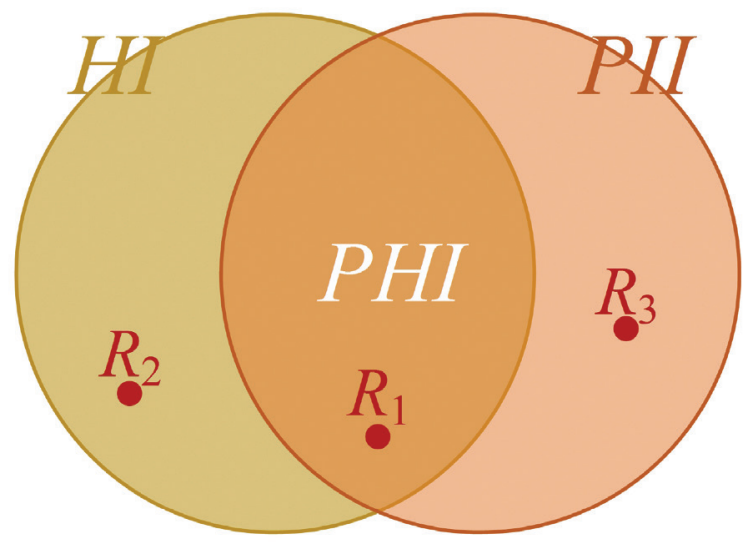

FIG. 1c. Protected health information $(P H I)$ is the intersection of health information $(H I)$ and personally identifying information (PII). Members of all sets are compound information such as $R_{1}$ a clinical report with personal identifiers, $R_{2}$ a de-identified clinical report, and $R_{3}$ a table of personal identifiers with no clinical connections. 1a is elementary information (e.g., first name $=$ "John", age $=$ "35"), whereas elements of the sets in Figure 1c are compound information (i.e., any combination of elementary information); hence, a record is a member of those sets in Figure 1c. A health record is PHI, only if it contains elements from both $H$ and PID (see the orange area in Figure 1c). Note that some seemingly elementary information such as "hospital admission date" may be a set of compound information [e.g., $\left\{h_{i}, d_{j}\right\}=\{$ "admitted to the hospital", "on June 1, 2017”\}].

The U.S. Public Health law supports federally funded studies on sensitive health issues such as sexual attitudes, sexually transmitted diseases, addictions, mental health, and illegal behaviors, by protecting PHI of those research subjects. Researchers of such studies may apply to the National Institutes of Health (NIH) to receive Certificates of Confidentiality (27). Researchers with certificates of confidentiality can disclose neither PHI nor PII of the research subjects collected for that particular study to "any Federal, State, or local civil, criminal, administrative, legislative, or other proceeding" even after the federal funding is concluded. The disclosure of PHI by researchers would only be allowed under certain conditions that have been specifically permitted by the human subject in his/ her informed consent (28).

\section{HIPAA Privacy Rule}

In 1996, the U.S. Congress enacted the Health Insurance Portability and Accountability Act (HIPAA) and required the Secretary of Health and Human Services (HHS) to promulgate standards to address. "(1) The rights that an individual who is a subject of individually identifiable $\mathrm{HI}$ should have. (2) The procedures that should be established for the exercise of such rights. (3) The uses and disclosures of such information that should be authorized or required" (29). In 1999, HHS proposed the initial version of the Privacy Rule as a set of privacy protection standards for handling and transmitting HI of individuals (30). The current version of the rule incorporated amendments of the Health Information Technology for Economic and Clinical Health (HITECH) Act and the Genetic Information Nondiscrimination (GINA) Act of 2008 (23).

The Privacy Rule prohibits selling PHI (31) or using it for marketing purposes unless a written authorization is obtained from the individual for that specific purpose. If the remuneration is received by the selling/marketing party, it must be explicitly stated (32).

PHI can be used by the provider for the purpose of the individual's care (i.e., for primary use) and disclosed to other providers for the same purpose or disclosed to health insurance services for payment notifications. PHI can also be disclosed to another person (e.g., a family member) designated by the 
individual. The individual has the right to be informed prior to any such disclosure and to restrict those disclosures. Upon the death of the individual, the provider can disclose PHI to a family member or designated person unless the individual had a request against such disclosures (33).

PHI can also be used or disclosed for secondary (non-care related) use in limited circumstances. If legally required, the provider may disclose PHI without the individual's authorization to a public health or government authority, to authorized programs related to workers' compensation, or to organizations involved in tissue/organ banking or transplantations (34).

Disclosures must always be limited to the minimum PHI that is "necessary to accomplish the intended purpose of the use, disclosure, or request" (31). Organizations can allow employee access only to those pieces of PHI that are appropriate and necessary to perform their duties (35). For example, a hospital registrar may access patients' names and addresses but not their diagnostic codes or clinical reports.

\section{Privacy rule for research}

Researchers can use PHI if they receive authorizations from the subjects of PHI. Without such authorization, also called informed consent, (36-38) researchers must apply to the institutional review board (IRB) for a waiver of authorization (22). The minimum necessary PHI can be released to researchers without prior authorization from the individual upon the approval of a waiver of authorization by an IRB or a privacy board $(31,34,35)$. Healthcare institutes can grant researchers access to PHI without an IRB approval in two limited cases: (a) The data can be disclosed to researchers if PHI belong to deceased individuals, (b) If the request is limited to reviewing PHI for a preparatory study to research and no PHI leaves the institute (34).

Waivers of authorization can be provided by IRBs only if the requested PHI is necessary for the study, the proposed study is deemed scientifically sound and important, the protocol is well planned, and all necessary safety, security and privacy measures are taken. Furthermore, researchers must demonstrate that;

1. "The research involves no more than minimal risk to the subjects.

2. The waiver or alteration will not adversely affect the rights and welfare of the subjects.

3. The research could not practicably be carried out without the waiver or alteration, and

4. Whenever appropriate, the subjects will be provided with additional pertinent information after

participation" (36).

Unless researchers substantiate why all PII elements preserved in the PHI are necessary, PII elements must be removed from the data prior to disclosing it to the study (31). The process of removing PII elements from PHI is known as de-identification.

\section{De-identification}

The Privacy Rule provides two different de-identification methods (Figure 2) $(23,39)$. In the first method, expert determination, an expert de-identifies PHI, documents the methodology, and quantifies the minimal risk of re-identification using generally accepted statistical and scientific methods.

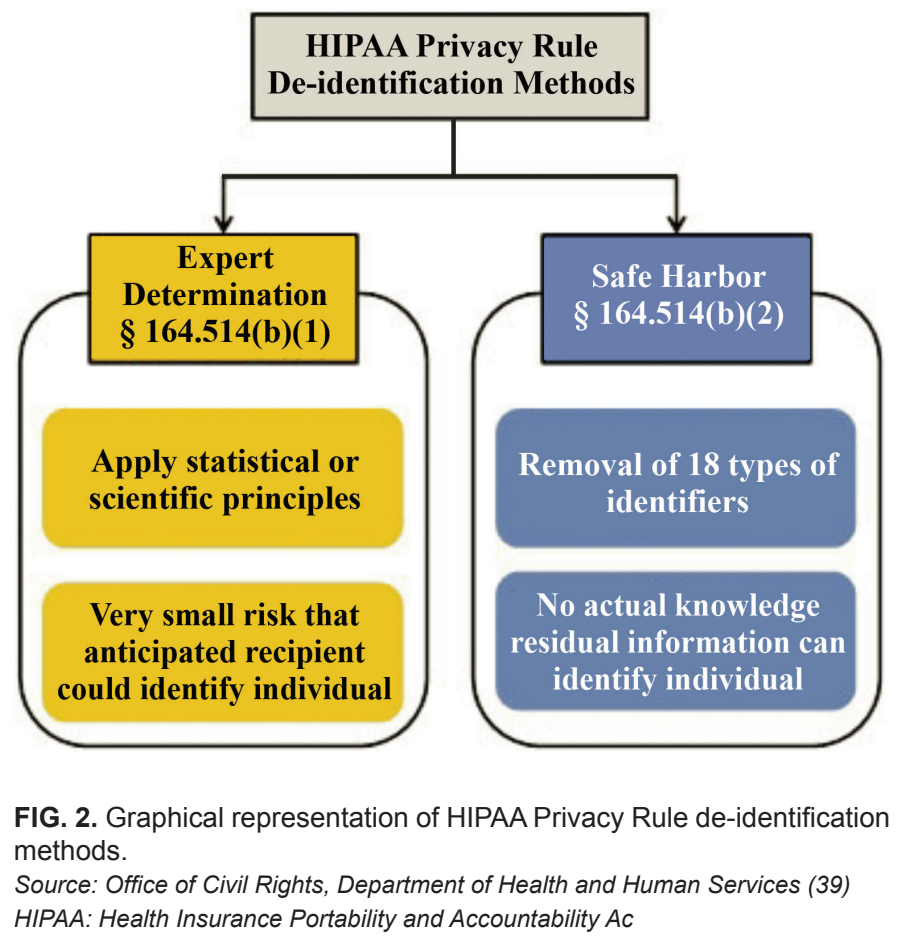

The second one called the Safe Harbor method requires 18 types of identifiers (PII elements) to be removed from data (Table 1). If the de-identifying party is aware of other PII elements that remained in the de-identified data and could identify the individual, they should be removed as well.

Demographic information most frequently used in clinical studies such as age, gender, ethnic origin and occupation are not included in the set of 18 PII elements. The exception is the category of ages 90 and above, which the Privacy Rule requires to be combined into a single age category, because in a given location, the size of the population at this particular age can be so small that the age information by itself can be used to identify the individual.

The Privacy Rule establishes a specific provision called Limited Data Set (LDS) when one or more of the 18 PII elements are required for a study. Since LDS would contain PII elements, the resulting $\mathrm{HI}$ is considered $\mathrm{PHI}$, but institutes are allowed to 
TABLE 1. Per HIPAA Privacy Rule, the following identifiers must be removed from PHI to obtain fully de-identify health information (*). As of 2010 , there were 18 sets of zip codes with distinct initial three digits whose corresponding population sizes were less than or equal to $20.000(60)$

1. Names,

2. All geographic subdivisions smaller than a state, except the first two digits of the zip code of the postal address. The third digit of the zip code can also be left intact, only if the size of the population in the area of the censored two digits is greater than 20.000 according to the most recent census data $(*)$,

3. All elements of dates (except year) for dates directly related to an individual, including birth date, admission date, discharge date, date of death; and all ages over 89 and all elements of dates (including year) indicative of such age, except that such ages and elements may be aggregated into a single category of age 90 or older,

4. Telephone numbers,

5. Fax number,

6. Electronic mail addresses,

7. Social security numbers,

8. Medical record numbers,

9. Health plan beneficiary numbers,

10. Account numbers,

11. Certificate/license numbers,

12. Vehicle identifiers and serial numbers, including license plate numbers,

13. Device identifiers and serial numbers,

14. Web universal resource locators (URLs),

15. Internet Protocol address numbers,

16. Biometric identifiers, including fingerprints and voiceprints,

17. Full-face photographic images and any comparable images,

18. Any other unique identifying number, characteristic, or code, except the ones that may be generated by the covered entity for re-identification.

disclose LDS to researchers without IRB review if researchers sign a data use agreement established by the institute.

If researchers can demonstrate that their study requires the following PII elements for research purposes, they can obtain this information within an LDS:

1. All date elements (i.e., no longer limited to year information, incl. birth date) and age information (incl. 90 and above),

2. Full ZIP code, town, city, and county information.

Expert Determination method is usually offered by commercial de-identification services with the claim that the Safe Harbor method removes necessary demographic and date information (40). Such claims hold no water given that within the framework of LDS, researchers can access all such information without an IRB review if and when they can demonstrate that such information is necessary for the study.

\section{De-identification of different data types}

There are four types of data: tabular, image/video, signal, and text data. De-identifying tabularly structured data is straightforward if specifications of the fields are defined. In most databases, records are represented in lines and fields in columns. If a particular column contains one of the $18 \mathrm{PII}$ elements, we can easily redact the contents of that column for all records. Genomic data is tabular in nature.

Privacy Rule dictates that full-face photographic images and images that can identify the individual should be removed, but it is possible to remove only the facial characteristics from pictures. For example, the faces of all individuals presented in Google Street View images (41-44) are blurred as are the letters and numbers on license plates of motor vehicles. To accomplish this task with images, one needs to use face and text recognition applications $(45,46)$.

DICOM is the most prevalent standard to represent and transmit clinical image data. Each DICOM image dataset comprises a header of structured (tabular) data and pixels of images. DICOM images can be de-identified mainly by deidentifying the tabular data section only; however, there are deidentification tools that recognize and de-identify "burned-in annotations" (46) or blur the facial characteristics on brain MR images, without any distortion of clinical image information of the brain (45).

Most signal data such as electrocardiography and electroencephalography do not require de-identification. The only signal data required to be de-identified is voiceprints, which are graphical representations of individual voices in terms of frequency, amplitude and duration. Through mathematical analysis, individuals can be re-identified from their voiceprints; thus, they are considered biometric identifiers like fingerprints. Genomic data is also unique to every individual. It is possible to detect a particular genomic sequence (of an individual) in a large collection of genomic sequences (47). In this sense, the nature of genomic data is similar to fingerprints. Note, however, neither gene sequences nor fingerprints by themselves can identify the individual. To use fingerprints for identification, an external information source, mapping each particular fingerprint to a personal name and other identifiers of that person, must be available. The only successful method in the literature for the re-identification of individuals whose genomic data were stored in a public site was using not the genomic data but the birthdate, full ZIP code and gender information (48). By removing all unnecessary demographic, date and geographic information from genomic databases and minimizing essential demographic information (e.g., by aggregating age information), the risk of a privacy breach can be greatly reduced. If a breach does happen, it would unlikely occur during the study of genomic data, but rather via the disclosure of other information mapping the specimen number or the genomic data to standard identifiers (i.e., PII) of the individual.

Text data is a much larger component of the electronic health record (EHR) than tabular data (49-52). Clinical narratives can provide indispensable information to research studies about the 
patient's clinical condition that other data types cannot (53). However, de-identifying free text data is more complicated than de-identifying tabular data because there is no schema similar to what databases have and no well-defined structure of records and fields. With no controlled vocabulary, expressions in English or in any other natural language are intrinsically vague such that the same word (e.g., "may") can have multiple meanings in different contexts; thus, distinguishing health information from PII can be challenging.

Still it is feasible to manually de-identify clinical reports if the amount of text is limited and if experienced de-identification professionals are available and well trained. In the era of Big Data, the first premise is rarely applicable. Manual deidentification can be very expensive for institutions and would become infeasible as the number of studies relying on clinical reports increases. Data providers may have little or no incentive to perform manual de-identification. Although they may charge only a nominal fee for such a service, by law, providers cannot seek any profit by providing the data. To provide manual de-identification services, institutes would have to undertake huge burdens of operational and financial overhead as well as risk negative publicity if the process failed.

\section{Types of automatic text de-identification systems}

Fortunately, there are automatic clinical text de-identification tools whose sensitivity and accuracy increase continuously. PARAT (54) and De-IDTM (55) are two such tools that are commercially available. There are also other freely available software applications such as MIST (56), de-id (57), and National Library of Medicine (NLM)-Scrubber (58-60) developed at MITRE, MIT and NIH, respectively. Both MIST and de-id have been academic exercises without a long-term plan for improvement and maintenance.

NLM-Scrubber was developed with a long-term goal of providing a non-commercial solution to biomedical scientists and institutions that do not have necessary resources to undertake the clinical text de-identification process. As a government research organization, NIH has no profit motive. The project aims to provide US taxpayers the best patient privacy protection and allows them to benefit from rapid scientific advances.

Current de-identification systems use various methods to recognize PII in clinical data, but neither a survey (61-64) of current de-identification systems nor computational techniques and mathematics of de-identification is within the scope of this review. However, clinical data scientists need to know what to expect from a de-identification system, how it can be used, and most critically, what types of input they need to provide the system to receive the desired output.

\section{Annotated health information}

Some clinical text de-identification systems such as MIST use supervised machine learning (ML) methods and require a set of training data where each PII element is annotated manually. Such ML systems either come with an annotation tool or are capable of using outputs of existing annotation tools (65). Annotation is a precursor of de-identification performed by human experts (66); thus, an ML system learns from the annotations of human experts,

and attempts to recognize PII elements in the non-training data and to replicate the human performance during the deidentification process. Other de-identification systems such as NLM-Scrubber operate without training data.

All health institutes that de-identify health records need to employ human experts to annotate a small subset of randomly selected health records from the data of the larger cohort that needs to be de-identified. This small set of annotated HI would serve as the gold standard to evaluate the performance of the automatic de-identification system of the institute $(67,68)$. Without such an evaluation and verification, a health institute would not know if the output of the de-identification system is truly de-identified.

An ML system requires annotated training data that is usually much larger than the annotated gold standard data. Unfortunately annotated gold standard data cannot be reused for training purposes since the two need to be mutually exclusive; otherwise, the evaluation results would be misleading. The size of the training data depends on the learning ability of the system and on the complexity of the data that needs to be de-identified; thus, an institute has to train the system in iterative steps with increasing size of training data, until the size increase does not significantly improve the system's performance. Due to the open-ended nature of the training data production and the large size of the prerequisite training data, the overhead of creating training datasets may be overwhelming for health institutes that lack the necessary human resources to carry out the task.

\section{Modes of de-identification}

From the clinical data scientist point of view, an automatic clinical text de-identification application is a black box; that is, the application takes some input and produces de-identified data - the underlying mechanism of de-identification does not matter much as long as the application produces the desired output. To produce optimal results, the data scientist needs to know the various operation modes available for the deidentification system in hand.

An earlier study (69) distinguished eight modes of deidentification, to which we add a ninth, pseudonymization 
(Table 2). These modes define how the user can operate a given de-identification system if the system provides a particular functionality. The first three change the mode of operation in terms of de-identification time and input. The next two alter the input and output modes, respectively. The following three modes involve different stakeholders as active participants in the de-identification operation, and the last mode moves de-identification to the cloud. Most of these modes can be combined to maximize the protection of patient privacy and the integrity of the de-identified data.

TABLE 2. Modes of de-identification

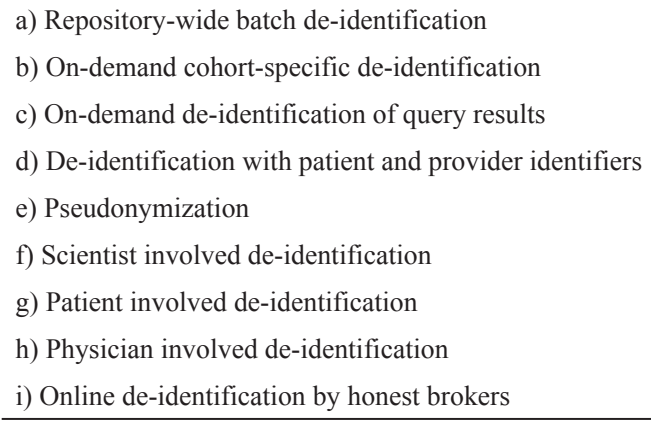

Repository-wide batch de-identification is the default mode of operation adopted by most (if not all) existing systems. For an institute, it is tempting to de-identify its entire repository at once and make the de-identified data available to researchers when requested. In contrast, the next two modes de-identify data on demand. The repository-wide batch mode makes the data available at the time of request without additional operational overhead. However, the data might have been deidentified using an older technology with a lower quality of de-identification; the de-identified data may be incomplete and/or incorrect if the source data has been updated since the de-identification occurred; and it may not contain some of the required demographic information necessary for the study.

In on-demand cohort-specific de-identification, the data of the cohort that researchers defined is de-identified on demand. Since modern de-identification systems are very fast, the delay between the data collection and de-identification would be insignificant. On-demand de-identification of query results requires the integration of the de-identification system into the EHR system. Results of the query can be de-identified on the fly before being displayed to researchers.

By augmenting the input mode of de-identification with patient and provider identifiers, the accuracy of results can be improved significantly (58). In the pseudonymization mode, the deidentified data replaces PII elements (e.g., "Fred Jones") with pseudonyms (e.g., "John Doe") instead of with a label of the corresponding PII element (e.g., "[Personal Name]"), so if the system fails to de-identify some PII elements, the user might not be aware of the failure as remaining PII elements blend in among other pseudonyms (70).

In the scientist involved de-identification mode, scientists actively participate in the de-identification, producing better de-identification results. If the scientist's active participation is ensured, the sensitivity of the de-identification system for recognizing PII elements can be increased manually. As a side effect of the increased sensitivity, some $\mathrm{HI}$ could be misidentified as PII. By reviewing the first batch of de-identified results, the scientist can identify a set of misidentified terms, which can then be input to the system, so those terms can be preserved during the second de-identification cycle. De-identification using this mode results in better protection of patient privacy and a more complete set of de-identified data with higher scientific value and data integrity.

Patient involved de-identification is hypothetical since no existing system currently offers patients to annotate their own records for de-identification purposes. In very rare occasions, the context of the narrative might inadvertently reveal the identity of the patient; e.g., "injured during his US championship match today" (71). In such cases, manual patient annotations would help improve de-identification results. Furthermore, as de-identified clinical reports become widely available to researchers, it is likely that patients would demand to be informed of which portions of their records are made available to researchers.

Physicians are occasionally required to cite the patient's full name and medical record number to link the record to that specific patient but it is a generally unnecessary and unadvisable practice. It would be best if medical students are trained to write anonymous clinical reports without patient identifiers so that these reports can be used for scientific research purposes in the future. Using physician involved de-identification mode, the system warns physicians whenever they use patient identifiers. If such identifiers are necessary for clinical care purposes, they can be automatically labeled and those labels then verified by the physician.

As big health data becomes widely available to clinical scientists, it will likely be accumulated and accessed at large centers such as state cancer registries, state universities, and government research centers, which can allocate the expertise and necessary resources to handle big data and provide services to other institutes nationwide. The online de-identification mode would enable scientists of smaller institutes to access de-identified data of much larger cohorts. Centers holding big health data can act as honest brokers, de-identify the data, develop proper data use agreements, and monitor compliance of users. 


\section{DISCUSSION}

Protecting patient privacy requires various technical tools. It involves regulations for sharing, de-identifying, securely storing, transmitting and handling PHI. It involves privacy laws and legal agreements. It requires establishing rules for monitoring privacy leaks, determining actions when they occur, and handling de-identified clinical narrative reports. Deidentification is one such indispensable instrument in this set of privacy tools.

Protecting patient privacy requires collaboration among all stakeholders, which include patients, PHI holding institutions, users of HI, developers of automatic de-identification tools, and regulatory and law enforcement government agencies. Each group has a different set of roles and responsibilities. For example, institutions should be held responsible to select the right tools, monitor the adequacy of these tools over time, and ensure the quality and content of de-identified data before presenting it to the user. They also are required to use these tools properly by supplying all necessary input to the deidentification system and utilizing all available modes of deidentification to maximize privacy protection. Institutions are also responsible to establish proper data use agreements.

Institutions and users of $\mathrm{HI}$ are equally responsible for ensuring that the requested and granted data comprise only the HI that is necessary for the study. Both regulatory agencies and institutions should empower patients to actively protect their privacy by monitoring their EHRs and let them know what portions of their data have been shared, with whom, and to what end. Institutions should demand from their users to provide study terms of interest to input to the de-identification process, so that the scientific integrity of the data can be preserved while privacy protection can be achieved at the highest level of sensitivity for de-identifying PHI.

As outlined above, the demand from institutions holding PHI is significant. Smaller institutions can be overwhelmed by the operational and financial overhead. There is little or no incentive structure for these institutions to take this challenge eagerly and share the data for secondary scientific use, particularly with scientists outside of those institutions. The entire scientific community including journal editors and the public, with the help of regulatory and grant providing agencies, should build incentive structures to support these institutions and make their contributions to the advancement of science visible.

In conclusion, Big Data makes the problem of patient privacy protection bigger and more difficult to attain; however, recent advances in computational de-identification help remedy the problem and enable scientists to access big health data by minimizing the risk to patient privacy. We have made great strides in developing both regulatory and technical privacy tools for the era of big data; however, this is still a work in progress. We reviewed the progress of patient privacy protection with a focus on the U.S. As seen in references, regulations have been continuously updated with numerous amendments. We did not discuss the European efforts but the regulations there are more in flux. In 2016, the European Parliament enacted the General Data Protection Regulation (GDPR), which will take effect in 2018 (38). GDPR provides patient privacy protection using a language similar to the Privacy Rule.

Thanks to the digital communication revolution, the world gets smaller every day. As everyone deserves to equally benefit from scientific advances, it is inevitable that any legal differences among nations including U.S., Europe, Canada, and Australia will soon be ironed out so that we all can collaborate to find cures to today's incurable diseases and improve the quality of life around the world.

Financial Disclosure: This work was supported by the Intramural Research Program of the NIH, National Library of Medicine. The author is the principal investigator in NLMScrubber project at NIH. He receives royalties from University of Pittsburgh for his contribution to a de-identification project; the resulting product was acquired by a third party, which today is known as De-ID Data Corp. NLM's Ethics Office reviewed and approved his appointment.

Conflict of Interest: No conflict of interest was declared by the author.

\section{REFERENCES}

1. Hippocrates. Jusjurandum (The Oath). In: Jones WHS, editor. Loeb Classical Library. Reprint: Hippocrates Collected Works I. Hippocrates ed. Cambridge, MA: Harvard University Press; 1868.

2. Higgins GL. The history of confidentiality in medicine: the physicianpatient relationship. Can Fam Physician 1989;35:921-6.

3. Parent WA. Recent work on the concept of privacy. Am Philos Q 1983;20:341-55.

4. Heins M. "The Right to Be Let Alone": Privacy and Anonymity at the U.S. Supreme Court. Revue Française D’études Américaines 2010:54-72.

5. Coke E. Semayne's case. In: Court of King's Bench, editor. 5 Co Rep 91a, 77 Eng Rep 1941604.

6. U.S. Constitution Amendment IV-search and seizure (1791).

7. Warren SD, Brandeis LD. The right to privacy. Harvard Law Review 1890;4:193-220.

8. Coleman AH. The Patient's Right to Privacy. J Natl Med Assoc 1961;53:207.

9. Al-Fedaghi SS. The "right to be let alone" and private information. In: Chen CS, Filipe J, Seruca I, Cordeiro J, editors. Enterprise Information Systems VII. Dordrecht: Springer Netherlands; 2006. p. 157-66.

10. Yamamoto R. [Management system of personal data protection in the health care field]. Rinsho Byori 2014;62:1129-34.

11. Universal declaration of human rights. United Nations (1948). 
12. Thomson JJ. The right to privacy. Philosophy and Public Affairs 1975;4:295-314.

13. Feinberg W. Recent developments in the law of privacy. Columbia Law Review 1948;48:713-31.

14. Veal WR. Torts-right of privacy. Louisiana Law Review 1949;9:17.

15. Thompson IE. The nature of confidentiality. J Med Ethics 1979;5:57-64.

16. Scanlon T. Thomson on privacy. Philosophy and Public Affairs 1975;4:31522.

17. Richards NM, Solove DJ. Privacy's Other Path: Recovering the law of confidentiality. Geo L J 2007;96:123-82.

18. Beardsley EL. Privacy: Autonomy and selective disclosure. In: Pennock JR, Chapman JW, editors. Privacy Vol XIII. Atherton Press; 1971:5670.

19. Code of medical ethics. American Medical Association (2001).

20. Directorate-General for Research and Innovation. Ethics for researchers, facilitating research excellence in FP7. Luxembourg: European Commission; 2013 (http://ec.europa.eu/research/participants/data/ref/ fp7/89888/ethics-for-researchers_en.pdf).

21. Bernasek A. Should tax bills be public information? The New York Times. 2010 Feb 13;Sect. Your Taxes.

22. Basic HHS policy for protection of human research subjects, 45 C.F.R. Sect. 46.102 (2017).

23. Sebelius K. 45 CFR Parts 160 and 164. Modifications to the HIPAA privacy, security, enforcement, and breach notification rules under the health information technology for Economic and Clinical Health Act and the Genetic Information Nondiscrimination Act; other modifications to the HIPAA rules; final rule In: Office of the Secretary of the Department of Health and Human Services, editor. Federal Register, Volume 78, No 172013. p. 5566-702.

24. Administrative simplifications: definitions, 42 U.S. Code Sect. 1320d (1996).

25. 45 CFR §160.103 Definitions. [ 65 FR 82798, Dec. 28, 2000, as amended at 67 FR 38019, May 31, 2002; 67 FR 53266, Aug. 14, 2002; 68 FR 8374, Feb. 20, 2003; 71 FR 8424, Feb. 16, 2006; 76 FR 40495, July 8, 2011; 77 FR 1589, Jan. 10, 2012; 78 FR 5687, Jan. 25, 2013]: Department of Health and Human Services; 2013.

26. Family educational and privacy rights, 20 U.S. Code Sect. 1232g (2010).

27. National Institutes of Health. Certificates of confidentiality ( $\mathrm{CoC})$ [7/12/2017]. Available from: https://humansubjects.nih.gov/coc/index, https://humansubjects.nih.gov/coc/faqs.

28. Research and investigations generally, 42 U.S. Code Sect. 241 (2016).

29. Health Insurance Portability and Accountability Act of 1996, Pub. L. No. 104-91 (August 21, 1996).

30. Office of Civil Rights. The HIPAA privacy rule 2015. Available from: https://www.hhs.gov/hipaa/for-professionals/privacy/index.html.

31. 45 CFR $\S 164.502$ Uses and disclosures of protected health information: general rules. [65 FR 82802, Dec. 28, 2000, as amended at 67 FR 53267, Aug. 14, 2002; 78 FR 5696, Jan. 25, 2013]: Department of Health and Human Services; 2013.

32. 45 CFR $\$ 164.508$ Uses and disclosures for which an authorization is required. [67 FR 53268, Aug. 14, 2002, as amended at 78 FR 5699, Jan. 25, 2013]: Department of Health and Human Services; 2013.

33. 45 CFR $\S 164.510$ Uses and disclosures requiring an opportunity for the individual to agree or to object. [ 65 FR 82802, Dec. 28, 2000, as amended at 67 FR 53270, Aug. 14, 2002; 78 FR 5699, Jan. 25, 2013]: Department of Health and Human Services; 2013.

34. 45 CFR $\$ 164.512$ Uses and disclosures for which an authorization or opportunity to agree or object is not required. [ 65 FR 82802, Dec. 28, 2000, as amended at 67 FR 53270, Aug. 14, 2002; 78 FR 5699, Jan. 25, 2013; 78 FR 34266, June 7, 2013; 81 FR 395, Jan. 6, 2016]: Department of Health and Human Services; 2016.
35. 45 CFR $\S 164.514$ Other requirements relating to uses and disclosures of protected health information. [ 65 FR 82802, Dec. 28, 2000, as amended at 67 FR 53270, Aug. 14, 2002; 78 FR 5700, Jan. 25, 2013; 78 FR 34266, June 7, 2013]: Department of Health and Human Services; 2013.

36. 45 CFR $\S 46.116$. General requirements for informed consent. [ 56 FR 28012, 28022, June 18, 1991, as amended at 70 FR 36328, June 23, 2005]: Department of Health and Human Services; 2005.

37. 21 CFR $\$ 50.20$ General requirements for informed consent. [ 46 FR 8951, Jan. 27, 1981, as amended at 64 FR 10942, Mar. 8, 1999]: Department of Health and Human Services; 1999.

38. The European Parliament and the Council of the European Union. General Data Protection Regulation. 2012/0011 (COD). Brussels Council of the European Union; 2016.

39. Office of Civil Rights. Guidance regarding methods for de-identification of protected health information in accordance with Health Insurance Portability and Accountability Act (HIPAA) privacy rule. In: U.S. Department of Health and Human Services, editor. 2012.

40. Privacy Analytics. When is it appropriate to use safe harbor? 2015 [7/9/2017]. Available from: https://privacy-analytics.com/de-id-university/ blog/using-safe-harbor-de-identification/.

41. Shankland S. Google begins blurring faces in Street View. c|net. 5/13/2008. Available from: https://www.cnet.com/news/google-begins-blurring-facesin-street-view/

42. Blake H. Google's EU warning over Street View privacy. The Telegraph. $2010 \mathrm{Feb} 26$.

43. Miller CC, O'Brien KJ. Germany's complicated relationship with Google Street View. The New York Times. 2013 Apr 23.

44. Johnston C. Google Street View's beefed-up privacy blurs cow's face. The Guardian. 2016 Sep 18.

45. Bischoff-Grethe A, Ozyurt IB, Busa E, Quinn BT, Fennema-Notestine C, Clark CP, et al. A technique for the deidentification of structural brain MR images. Hum Brain Mapp 2007;28:892-903.

46. González DR, Carpenter T, van Hemert JI, Wardlaw J. An open source toolkit for medical imaging de-identification. Eur Radiol 2010;20:1896904.

47. Homer N, Szelinger S, Redman M, Duggan D, Tembe W, Muehling J, et al. Resolving individuals contributing trace amounts of DNA to highly complex mixtures using high-density SNP genotyping microarrays. PLoS Genet 2008;4:e1000167.

48. Sweeney L, Abu A, Winn J. Identifying participants in the Personal Genome Project by name. Data Privacy Lab, IQSS, Harvard University. [White paper]. In press 2013.

49. van 't Hoff E. The data explosion along the care cycle. NVKVV 16de Colloquim ICT en gezondheidszorg; Affligem, Belgium: Dell Healthcare; 2012.

50. Wu W, Ding H. Big data solutions for healthcare. [Presentation]. In press 2013.

51. Datamark Inc. Unstructured data in electronic health record (EHR) systems: challenges and solutions. 2013.

52. Rhinehart C. The impact of cognitive computing on healthcare. IBM Watson Health; 2015.

53. Johnson SB, Bakken S, Dine D, Hyun S, Mendonça E, Morrison F, et al. An electronic health record based on structured narrative. J Am Med Inform Assoc 2008;15:54-64.

54. Privacy Analytics. PARAT maintenance and support information [updated 6/25/20147/9/2017]. Available from: http://knowledgebase.privacyanalytics.com/index.php?/article/AA-00335/25/PARAT/General/PARATMaintenance-and-Support-Information.html.

55. Gupta D, Saul M, Gilbertson J. Evaluation of a deidentification (De-Id) software engine to share pathology reports and clinical documents for research. Am J Clin Pathol 2004;121:176-86. 
56. Wellner B, Huyck M, Mardis S, Aberdeen J, Morgan A, Peshkin L, et al. Rapidly retargetable approaches to de-identification in medical records. J Am Med Inform Assoc 2007;14:564-73.

57. Neamatullah I, Douglass M, Lehman L-w, Reisner A, Villarroel M, Long $\mathrm{W}$, et al. Automated de-identification of free-text medical records. BMC Med Inform Decis Mak 2008;8:32.

58. Kayaalp M, Browne AC, Callaghan FM, Dodd ZA, Divita G, Ozturk S, et al. The pattern of name tokens in narrative clinical text and a comparison of five systems for redacting them. J Am Med Inform Assoc 2014;21:423-31.

59. Kayaalp M, Browne AC, Dodd ZA, Sagan P, McDonald CJ. Clinical text de-identification research. A report to the Board of Scientific Counselors. U.S. National Library of Medicine, National Institutes of Health, Communications LHNCfB; 2013. Report No 2013-001.

60. Kayaalp M, Browne AC, Dodd ZA, Sagan P, McDonald CJ. Deidentification of Address, Date, and Alphanumeric Identifiers in Narrative Clinical Reports. AMIA Annu Symp Proc 2014:767-76.

61. Uzuner O, Luo Y, Szolovits P. Evaluating the state-of-the-art in automatic de-identification. J Am Med Inform Assoc 2007;14:550-63.

62. Meystre SM, Friedlin FJ, South BR, Shen S, Samore MH. Automatic deidentification of textual documents in the electronic health record: a review of recent research. BMC Med Res Methodol 2010;10:70.

63. Kushida CA, Nichols DA, Jadrnicek R, Miller R, Walsh JK, Griffin K. Strategies for de-identification and anonymization of electronic health record data for use in multicenter research studies. Med Care 2012;50 Suppl:S82-101.
64. Stubbs A, Kotfila C, Uzuner Ö. Automated systems for the de-identification of longitudinal clinical narratives: Overview of 2014 i2b2/UTHealth shared task Track 1. J Biomed Inform 2015;58 Supplement:S11-S9.

65. MITRE. Annotations [7/9/2017]. Available from: http://mist-deid. sourceforge.net/current_docs/html/annotation_intro.html.

66. Stubbs A, Uzuner O. De-identification of medical records through annotation. In: Ide N, Pustejovsky J, editors. Handbook of Linguistic Annotation. Dordrecht, The Netherlands: Springer; 2017:1433-59.

67. Browne AC, Kayaalp M, Dodd ZA, Sagan P, McDonald CJ. The Challenges of Creating a Gold Standard for De-identification Research. AMIA Annu Symp Proc 2014:353-8.

68. Kayaalp M, Browne AC, Sagan P, McGee T, McDonald CJ. Challenges and Insights in Using HIPAA Privacy Rule for Clinical Text Annotation. AMIA Annu Symp Proc 2015:707-16.

69. Kayaalp M. Modes of de-identification. Proc AMIA Annu Symp; Forthcoming; an advance copy available at https://hncbc.nlm.nih.gov/ publication/pub9526.

70. Carrell D, Malin B, Aberdeen J, Bayer S, Clark C, Wellner B, et al. Hiding in plain sight: use of realistic surrogates to reduce exposure of protected health information in clinical text. J Am Med Inform Assoc 2013;20:3428.

71. Kayaalp M, Sagan P, Jones JK, Browne AC, McDonald CJ. Guidelines for annotating personal identifiers in the clinical text repository of the National Institutes of Health. Available at https://scrubber.nlm.nih.gov/ annotation/ 